\title{
Self-Similar Universal Homogeneous Statistical Solutions of the Navier-Stokes Equations
}

\author{
C. Foias ${ }^{1}$ and R. Temam ${ }^{2}$ \\ 1 Department of Mathematics, Indiana University, Bloomington, IN 47405, USA \\ 2 Laboratoire d'Analyse Numérique, Batiment 425, Université Paris-Sud, 91405 Orsay, France
}

\begin{abstract}
In this note we consider a family of statistical solutions of the NavierStokes equations (i.e. time dependent solutions of the Hopf equation) which seem to constitute the rigorous mathematical framework for the theory of homogeneous turbulence [1], [13]. The main feature of these solutions is that they are the transforms under suitable scalings of the stationary statistical solutions of a new system of equations (the Eq. (2) below).
\end{abstract}

\section{Introduction}

The theory of fully developed turbulence is nearly universally believed to be essentially that of the evolution of statistical distributions of flows governed by the Navier-Stokes equations:

$$
\frac{\partial u}{\partial t}-v \Delta u+(u \cdot \nabla) u+\nabla p=0, \nabla \cdot u=0 .
$$

Although significant progress has been made in the last 15 years in the rigorous mathematical approach to this theory $[2,4,6,9,12,21, \ldots]$, no concrete family of homogeneous statistical solutions of the Navier-Stokes equations was found, nor is there as yet a consistent way of connecting these solutions with the Kolmogorov spectral estimates. In this paper we show that there exists a natural family of homogeneous statistical solutions of the Navier-Stokes equations enjoying some properties of self similarity and universality (Sect. 3 ). These solutions are obtained by suitable scalings of the stationary homogeneous statistical solutions of the equations

$$
\frac{\partial u}{\partial t}-\frac{1}{2} u-\frac{1}{2}(x \cdot \nabla) u-\Delta u+(u \cdot \nabla) u+\nabla q=0, \nabla \cdot u=0
$$

(see Sect. 5 below). Note that the stationary form of Eqs. (2) differs only slightly from the (still not well understood) equations

$$
\frac{1}{2} u+\frac{1}{2}(x \cdot \nabla) u-\Delta u+(u \cdot \nabla) u+\nabla q=0, \nabla \cdot u=0,
$$


introduced long ago by J. Leray [14] in connection with the problem of the spontaneous development of singularities for the solutions to the time dependent (deterministic) Navier-Stokes equations (see the comments in Sect. 3 below). Although we have not as yet proved an existence theorem for the type of solutions we need for (2), our results seem to be consistent with the conjecture that such solutions exist (see Sects. 6 and 8). As a byproduct it turns out that the Leray equations (3) do not have such solutions (i.e. stationary statistical solutions with some regularity properties, see the Remark in Sect. 6). The connection with the Kolmogorov spectrum is briefly discussed in Sect. 7 and Sect. 8.3, while in Sect. 8 we comment on the physical meaning of our present approach.

It seems to us that our present note constitutes a basis for a rigorous approach to the theory of fully developed turbulence as treated in the classical monographs G. K. Batchelor [1] and L. D. Landau-E. M. Lifshitz [13] or in A. J. Chorin [4]. Since this approach is essentially based on the Hopf equation [11], as considered in [9], it has no significant connection to the turbulence modelling theories, as for instance those presented in [4]. Further developments will appear elsewhere and in [7].

\section{Preliminary}

1.1. Let $L^{2}\left(\mathbb{R}^{3}\right)$ denote the space of (classes of) real functions which are square integrable on $\mathbb{R}^{3}$ with respect to the Lebesgue measure. For an integer $m \geqq 1$, let $H^{m}\left(\mathbb{R}^{3}\right)$ denote the Sobolev space of functions which, together with their derivatives of order $\leqq m$, belong to $L^{2}\left(\mathbb{R}^{3}\right)$. Endowed with the scalar products,

$$
\begin{aligned}
& (u, v)=\int_{\mathbb{R}^{3}} u(x) v(x) d x \text { for } u, v \in L^{2}\left(\mathbb{R}^{3}\right) \\
& (u, v)_{m}=\sum_{|\alpha| \leqq m}\left(D^{\alpha} u, D^{\alpha} v\right) \text { for } u, v \in H^{m}\left(\mathbb{R}^{3}\right)(m=1,2, \ldots) .
\end{aligned}
$$

Here $L^{2}\left(\mathbb{R}^{3}\right)$, and $H^{m}\left(\mathbb{R}^{3}\right)$ respectively, are Hilbert spaces. In the above

$$
\begin{aligned}
D^{\alpha} & =D_{1}^{\alpha_{1}} D_{2}^{\alpha_{2}} D_{3}^{\alpha_{3}}, D_{j}=\partial / \partial x_{j} \quad(j=1,2,3), \\
\alpha & =\left(\alpha_{1}, \alpha_{2}, \alpha_{3}\right) \in \mathbb{N}^{3} \text { and }|\alpha|=\alpha_{1}+\alpha_{2}+\alpha_{3} .
\end{aligned}
$$

We let also

$$
|u|=(u, u)^{1 / 2},|v|_{m}=(v, v)_{m}^{1 / 2} \text { for } u \in L^{2}\left(\mathbb{R}^{3}\right), v \in H^{m}\left(\mathbb{R}^{3}\right)
$$

and

$$
\left(\left(w_{1}, w_{2}\right)\right)=\sum_{j=1}^{3}\left(D_{j} w_{1}, D_{j} w_{2}\right),\|w\|=((w, w))^{1 / 2} \text { for } w, w_{1}, w_{2} \in H^{1}\left(\mathbb{R}^{3}\right) .
$$

The scalar products and the norms in $L^{2}\left(\mathbb{R}^{3}\right)^{3}$ or $H^{m}\left(\mathbb{R}^{3}\right)^{3}$ are defined by

$$
(u, v)=\sum_{j=1}^{3}\left(u_{j}, v_{j}\right), \text { respectively }(u, v)_{m}=\sum_{j=1}^{3}\left(u_{j}, v_{j}\right)_{m},
$$

where $u=\left(u_{1}, \ldots, u_{n}\right), v=\left(v_{1}, \ldots, v_{n}\right)$, etc... Endowed with these scalar products, $L^{2}(\mathbb{R})^{3}$ and $H^{m}\left(\mathbb{R}^{3}\right)^{3}$ will be denoted by $\mathbb{L}^{2}\left(\mathbb{R}^{3}\right)$ and $\mathbb{H}^{m}\left(\mathbb{R}^{3}\right)$ respectively. 
We denote by $\mathbb{L}_{\text {loc }}^{2}\left(\mathbb{R}^{3}\right)$, respectively $\mathbb{T}_{\text {loc }}^{m}\left(\mathbb{R}^{3}\right)$, the space of functions which locally (i.e. on any bounded measurable subset of $\mathbb{R}^{3}$ ) belong to $\mathbb{L}^{2}\left(\mathbb{R}^{3}\right)$, respectively to $\mathbb{H}^{m}\left(\mathbb{R}^{3}\right)$. For any bounded measurable subset $Q$ of $\mathbb{R}^{3}$ such that $|Q|=$ measure of $Q>0$, we set

$$
\begin{aligned}
& |u|_{Q}=\left(\frac{1}{|Q|_{Q}} \int_{Q}|u(x)|^{2} d x\right)^{1 / 2} \text { for } u \in \mathbb{L}_{\mathrm{loc}}^{2}\left(\mathbb{R}^{3}\right), \\
& \|v\|_{Q}=\left(\sum_{j=1}^{m}\left|D_{j} \cdot u\right|_{Q}^{2}\right)^{1 / 2}=\left(\frac{1}{|Q|} \int_{Q}|\nabla u(x)|^{2} d x\right)^{1 / 2} \text { for } v \in \mathbb{H}^{1}\left(\mathbb{R}^{3}\right),
\end{aligned}
$$

where $\nabla=\left(D_{1}, D_{2}, D_{3}\right)$ and $|\nabla u(x)|^{2}=\sum_{j=1}^{3}\left|\nabla u_{j}(x)\right|^{2}, x \in \mathbb{R}^{3}$.

Obviously, $\mathbb{L}_{\text {loc }}^{2}\left(\mathbb{R}^{3}\right)$ and $\mathbb{H}^{1}\left(\mathbb{B}^{3}\right)$ are Frechet spaces with respect to the families of semi-norms

$$
\left\{|u|_{Q}: Q=(a, b)^{3},-\infty<a<b<+\infty\right\}
$$

and

$$
\left\{\left(|u|_{Q}^{2}+\|u\|_{Q}^{2}\right)^{1 / 2}: Q=(a, b)^{3},-\infty<a<b<\infty\right\}
$$

respectively.

A basic role will be played by the subspaces

$$
\begin{aligned}
H_{\mathrm{loc}} & =\left\{u \in \mathbb{L}_{\mathrm{loc}}^{2}\left(\mathbb{R}^{3}\right), \nabla \cdot u=0\right\}, \\
V_{\mathrm{loc}} & =\left\{v \in \mathbb{H}_{\mathrm{loc}}^{1}\left(\mathbb{R}^{3}\right), \nabla \cdot v=0\right\},
\end{aligned}
$$

which are also Frechet spaces when endowed with the corresponding systems of semi-norms.

1.2. For $a \in \mathbb{R}^{3}$, we denote by $\tau_{a}$ the translation operator

$$
\left(\tau_{a} u\right)(x)=u(x-a), x \in \mathbb{R}^{3}
$$

on $H_{\text {loc }}$ and $V_{\text {loc }}$. A homogeneous measure on $H_{10 c}$ is, by definition, a Borel measure on $H_{1 \mathrm{loc}}$ such that

$$
\tau_{a}(\mu)=\mu \text { for all } a \in \mathbb{R}^{3}
$$

In the sequel all homogeneous measures on $H_{10 c}$ will be probability measures, i.e. positive and of total mass 1 . In this case the homogeneity of $\mu$ means that

$$
\int \Phi\left(\tau_{a} u\right) d \mu(u)=\int \Phi(u) d \mu(u)
$$

for all $a \in \mathbb{R}^{3}$ and $\Phi$ belonging to the space $B\left(H_{\text {loc }}\right)$ of the real bounded continuous functions on $H_{\text {loc }}$.

For a homogeneous measures $\mu$ on $H_{\text {loc }}$, the integrals

$$
\frac{1}{2} \int|u|_{Q}^{2} d \mu(u) \text { and } \int\|u\|_{Q}^{2} d \mu(u)
$$

are independent of $Q$, and they will be denoted by $e(\mu)$ and $E(\mu)$, respectively. 


\section{Homogeneous Statistical Solution}

2.1. By a Homogeneous Statistical Solution of the Navier-Stokes equations we mean a family $\left(\mu_{t}\right)_{0 \leqq t<\infty}$ of homogeneous (probability) measures on $H_{\text {loc }}$, such that

$$
\begin{gathered}
t \mapsto \int \Phi(u) d \mu_{t}(u) \text { is measurable (for all } \Phi \in B\left(H_{\mathrm{loc}}\right) \text { ), } \\
e\left(\mu_{t}\right)+v \int_{0}^{t} E\left(\mu_{s}\right) d s \leqq e\left(\mu_{0}\right) \text { for all } 0<t<\infty,
\end{gathered}
$$

and finally

$$
\begin{gathered}
\int \Phi(u) d \mu_{t}(u)+\int_{0}^{t} \int\left[v\left(\left(u, \Phi^{\prime}(u)\right)\right)+\left((u \cdot \nabla) u, \Phi^{\prime}(u)\right)\right] d \mu_{s}(u) d s \\
=\int \Phi(u) d \mu_{0}(u)
\end{gathered}
$$

for all $\Phi \in \mathscr{T}, \mathscr{T}$ being the class of all functions on $H_{\text {loc }}$ of the form

$$
\Phi(u)=\varphi\left(\left(u, g_{1}\right), \ldots,\left(u, g_{k}\right)\right) \text { for } u \in H_{\text {loc }},
$$

where $\varphi$ is a $\mathscr{C}^{1}$ function on $\mathbb{R}^{k}(k=1,2, \ldots)$ bounded together with its first derivatives, while

$$
g_{j} \in V_{c} \text { for all } j=1,2, \ldots, k \text {. }
$$

Here $V_{c}$ denotes the subset of elements of $V_{\text {loc }}$ (respectively $H_{\text {loc }}$ ) with compact support (in $\mathbb{R}^{n}$ ); and $\Phi^{\prime}$ denotes the differential of $\Phi$ in $H_{\text {loc }}$, i.e. $\Phi^{\prime}(u, v)=$ $(d / d \alpha) \Phi(u+\alpha v)_{\alpha=0}$ for all $v \in H_{c}$.

Let us recall that in (2.3), the coefficient $v>0$ is the (kinematic) viscosity of the fluid. Also let us recall the following fundamental fact related to the previous definition (see [9]; see also [22, 23]):

Theorem. For every homogeneous (probability) measure $\mu$ on $H_{1 \mathrm{loc}}$ such that $e(\mu)<\infty$, there exists a homogeneous statistical solutions of the Navier-Stokes equations $\left(\mu_{t}\right)_{0 \leqq t<\infty}$ such that $\mu_{0}=\mu$.

Obviously this theorem shows that the initial value problem for the homogeneous statistical solutions of the Navier-Stokes equations is solvable.

2.2. Finally let us agree that a stationary homogeneous statistical solution of Eqs. (2) is a homogeneous (probability) measure $\mu$ on $H_{\text {loc }}$ such that

$$
E(\mu)<\infty,
$$

and

$$
\int\left[\left(-\frac{1}{2} u-\frac{1}{2}(x \cdot \nabla) u, \Phi^{\prime}(u)\right)+\left(\left(u, \Phi^{\prime}(u)\right)\right)+\left((u \cdot \nabla) u, \Phi^{\prime}(u)\right)\right] d \mu(u)=0
$$

for all functions $\Phi \in \mathscr{T}^{1}$.

$1 \mathrm{By}$ virtue of the homogeneity of $\mu$, Eq. (2.7) is translation invariant (although the deterministic equations (2) are not translation invariant). Since for any $a \in \mathbb{R}^{3}$ and $\Phi \in \mathscr{T}$, we have

$$
\begin{aligned}
\int\left(a \cdot \nabla u, \Phi^{\prime}(u)\right) d \mu(u) & =+\int\left(a \cdot \nabla\left(\tau_{a} u\right), \Phi^{\prime}\left(\tau_{a} \mu\right)\right) d \mu(u) \\
& =-\left.\frac{d}{d s} \int \Phi\left(\tau_{a s} u\right) d \mu(u)\right|_{s=1}=0
\end{aligned}
$$


2.3. Comments. J. Leray has introduced Eqs. (3) in [14] in relation with the problem of the spontaneous development of singularities for the solutions to the time dependent deterministic Navier-Stokes equations when the dimension of space is 3 . Indeed if $U \in V_{\text {loc }} \cap \mathbb{H}^{1}\left(\mathbb{R}^{3}\right)$ is a weak solution of the Leray equations (3) and $t_{0}>0$, then

$$
u(x, t)= \begin{cases}\left(t_{0}-t\right)^{-1 / 2} U\left(x\left(t_{0}-t\right)^{-1 / 2}\right) & \text { for } 0<t<t_{0} \\ 0 & \text { for } t \geqq t_{0} .\end{cases}
$$

is a weak solution (in Leray's sense, nowadays in a classical sense) of the NavierStokes equations, such that

$$
\|u(\cdot, t)\|^{2}=\left(t_{0}-t\right)^{-1 / 2}\|U\|^{2} \text { for } 0<t<t_{0} .
$$

Therefore if such an $U \neq 0$ exists, one has a weak solution which is not globally regular (see [14]). The existence of such an $U$ is still an open problem, i.e. it has not yet been proved nor disproved. More generally the problem of the spontaneous appearance of singularities for the solutions to the time dependent Navier-Stokes equations in dimension 3 is still open; the reader is referred to the work of $V$. Scheffer [17-20], L. Cafarelli-R. Kohn-L. Nirenberg [3], and C. Foias-R. Temam [8], for the recent developments in this direction.

Similarly if $V \in V_{\text {loc }} \cap \mathbb{H}^{1}\left(\mathbb{R}^{n}\right)$ is a weak stationary solution of our Eqs. (2) and $t_{0}>0$, then

$$
u(x, t)= \begin{cases}0 & \text { for } 0<t \leqq t_{0} \\ \left(t-t_{0}\right)^{-1 / 2} V\left(x\left(t-t_{0}\right)^{-1 / 2}\right) & \text { for } t>t_{0}\end{cases}
$$

is again a weak solution of the Navier-Stokes equations. If $V \neq 0,(2.9)$ is a nonregular weak solution which invalidates the uniqueness of the initial value problem for the weak solutions of the Navier-Stokes equations. No such a $V$ is known, and more generally we recall that the problem of the uniqueness of the Hopf-Leray weak solutions to the time dependent deterministic Navier-Stokes equations in dimension 3 is still open.

\section{Self-Similar Universal Statistical Solutions}

3.1. First let us make some simple remarks concerning the changes of scale. For $u \in H_{\mathrm{loc}}$ and $\left.\xi, \lambda \in\right] 0, \infty[$ we set

$$
\left(\sigma_{\xi, \lambda} u\right)(x)=\xi u(\lambda x) \quad\left(\forall x \in \mathbb{R}^{3}\right) .
$$

Let moreover $\left(\mu_{t}\right)_{t \geqq 0}$ be a homogeneous statistical solution of the Navier-Stokes equations, let $\Phi \in \mathscr{T}$ and $\theta>0$. Then

$$
\begin{aligned}
& e\left(\sigma_{\xi, \lambda}\left(\mu_{t / \theta}\right)\right)=\frac{1}{2} \int|u|_{Q}^{2} d \sigma_{\xi, \lambda}\left(\mu_{t / \theta}\right)(u) \\
& \quad=\frac{1}{2} \int\left|\sigma_{\xi, \lambda} u\right|_{Q}^{2} d \mu_{t / \theta}(u)=\frac{1}{2} \int \xi^{2}|u|_{\lambda Q}^{2} d \mu_{t / \theta}(u) \\
& \quad=\xi^{2} e\left(\mu_{t / \theta}\right)
\end{aligned}
$$


and

$$
\begin{aligned}
& E\left(\sigma_{\xi, \lambda}\left(\mu_{t / \theta}\right)\right)=\int\|u\|_{Q}^{2} d \sigma_{\xi, \lambda}\left(\mu_{t / \theta}\right)(u) \\
& \quad=\int\left\|\sigma_{\xi, \lambda} u\right\|_{Q}^{2} d \mu_{t / \theta}(u)=\xi^{2} \int \frac{1}{|Q|} \int_{Q}\left|\nabla_{x}[u(\lambda x)]\right|^{2} d x d \mu_{t / \theta}(u) \\
& \quad=\xi^{2} \lambda^{2} \int \frac{1}{|Q|} \int_{Q}|(\nabla u)(\lambda x)|^{2} d x d \mu_{t / \theta}(u) \\
& =\xi^{2} \lambda^{2} \int\|u\|_{\lambda Q}^{2} d \mu_{t / \theta}(u)=\xi^{2} \lambda^{2} E\left(\mu_{t / \theta}\right) .
\end{aligned}
$$

Moreover we also have for any $\Phi \in \mathscr{T}^{2}$

$$
\begin{aligned}
\frac{d}{d t} \int & \Phi d \sigma_{\xi, \lambda}\left(\mu_{t / \theta}\right)=\frac{d}{d t} \int \Phi\left(\sigma_{\xi, \lambda} u\right) d \mu_{t / \theta}(u) \\
= & -\frac{1}{\theta} \int\left[v\left(\left(u, \nabla_{u}\left[\Phi\left(\sigma_{\xi, \lambda} u\right)\right]\right)\right)\right. \\
& \left.+\left((u \cdot \nabla) u, \nabla_{u}\left[\Phi\left(\sigma_{\xi, \lambda} u\right)\right]\right)\right] d \mu_{t / \theta}(u) \\
= & -\frac{1}{\theta} \int\left[v\left(\left(u, \frac{1}{\lambda^{3}} \sigma_{\xi, \lambda} \Phi^{\prime}\left(\sigma_{\xi, \lambda} u\right)\right)\right)\right. \\
& \left.+\left(\sigma_{\xi, \lambda}((u \cdot \nabla) u), \Phi^{\prime}\left(\sigma_{\xi, \lambda} u\right)\right)\right] d \mu_{t / \theta}(u) \\
=- & \frac{1}{\theta} \int\left[\frac{v}{\lambda^{2}}\left(\left(\sigma_{\xi, \lambda} u, \Phi^{\prime}\left(\sigma_{\xi, \lambda} u\right)\right)\right)\right. \\
& \left.+\frac{1}{\xi \lambda}\left(\left(\left(\sigma_{\xi, \lambda} u\right) \cdot \nabla\right)\left(\sigma_{\xi, \lambda} u\right), \Phi^{\prime}\left(\sigma_{\xi, \lambda} u\right)\right)\right] d \mu_{t / \theta}(u) \\
= & -\frac{1}{\theta \xi \lambda} \int\left[\frac{v \xi}{\lambda}\left(\left(u, \Phi^{\prime}(u)\right)\right)+\left((u \cdot \nabla) u, \Phi^{\prime}(u)\right)\right] d \sigma_{\xi, \lambda}\left(\mu_{t / \theta}\right)(u) .
\end{aligned}
$$

Thus we can now conclude with the following

Lemma 1. If $\left(\mu_{t}\right)_{0 \leqq t \leqq \infty}$ is a homogeneous statistical solution of the Navier-Stokes equations (with the viscosity $=v$ ), then the formula

$$
\tilde{\mu}_{t}=\sigma_{\xi, \lambda}\left(\mu_{t \xi \lambda}\right) \text { for } t \geqq 0
$$

defines a homogeneous statistical solution of the Navier-Stokes equations with viscosity

$$
\tilde{v}=v \xi / \lambda,
$$

$2 \nabla \Phi=\Phi^{\prime}$ represents the Frechet differential of a function $\Phi ; \nabla_{a}[\quad]$ represents the Frechet differential with respect to $a$ of the function of $a$ written between the brackets [ ] ( $a=$ some independent variable) 
such that

$$
\begin{aligned}
e\left(\tilde{\mu}_{t}\right) & =\xi^{2} e\left(\mu_{t \xi \lambda}\right) \\
E\left(\tilde{\mu}_{t}\right) & =\xi^{2} \lambda^{2} E\left(\mu_{t \xi \lambda}\right) .
\end{aligned}
$$

3.2. We can now state the main definitions of our discussion:

Definition 1. By a universal homogeneous statistical solution of the Navier-Stokes equations we mean a family $\left\{\mu^{v, \varepsilon}\right\}$ of homogeneous (probability) measures on $H_{\text {loc }}$ depending on two real parameters $v, \varepsilon>0$, such that

$$
e\left(\mu^{v, \varepsilon}\right)<\infty, \varepsilon=\nu E\left(\mu^{v, \varepsilon}\right),
$$

and for any $\mu=\mu^{v, \varepsilon_{0}}$ there exists a homogeneous statistical solution $\left(\mu_{t}\right)_{0 \leqq t<\infty}$ of the Navier-Stokes equations, with viscosity $v$, satisfying $\mu_{0}=\mu^{v, \varepsilon_{0}}$ and

$$
\mu_{t} \in\left\{\mu^{v, \varepsilon}: 0<\varepsilon<\infty\right\} \text { for all } t \geqq 0 .
$$

We shall moreover assume that this solution satisfies a stronger form of (2.2), namely the energy equation

$$
e\left(\mu_{t}\right)+v \int_{0}^{t} E\left(\mu_{s}\right) d s=e\left(\mu_{0}\right) \text { for all } t \geqq 0
$$

Definition 2. A universal homogeneous statistical solution of the Navier-Stokes equations is called self-similar if $\sigma_{\xi, \lambda}$ takes (for all $\xi, \lambda>0$ ) all the homogeneous statistical solutions considered in Definition 1 into solutions of the same kind.

A more precise content of this definition is given by the following

Proposition 1. Let $\left(\mu^{v, \varepsilon}\right)_{v, \varepsilon>0}$ be a self-similar universal statistical solution of the Navier-Stokes equations. Then

$$
\sigma_{\xi, \lambda}\left(\mu^{v, \varepsilon}\right)=\mu^{v \xi / \lambda, \xi^{3} \lambda \varepsilon}
$$

for all $\xi, \lambda, v, \varepsilon>0$.

Proof. If $\left(\mu_{t}\right)_{t>0}$ is any homogeneous statistical solution of the type considered in Definition 1, then, by (2.8) and (3.9) we have

$$
\mu_{t}=\mu^{v, \varepsilon(t)}, \text { where } \varepsilon(t)=\nu E\left(\mu_{t}\right)
$$

for all $t \geqq 0$. By Lemma $1,\left(\tilde{\mu}_{t}\right)_{t \geqq 0}$ is a homogeneous statistical solution of the NavierStokes equations with viscosity $\tilde{v}=v \xi / \lambda$. By Definition 2 , we have

$$
\tilde{\mu}_{t}=\mu^{\tilde{v}, \tilde{\varepsilon}(t)} \text {, where } \tilde{\varepsilon}(t)=\tilde{v} E\left(\tilde{\mu}_{t}\right)
$$

for all $t \geqq 0$. By (3.12), (3.6), (3.7) and (3.13) we have

$$
\dot{\varepsilon}(t)=\frac{v \xi}{\lambda} \xi^{2} \lambda^{2} E\left(\mu_{t \xi \lambda}\right)=\xi^{2} \lambda \varepsilon(t \xi \lambda) \text { for all } t \geqq 0,
$$

and in particular $\tilde{\varepsilon}(0)=\xi^{3} \lambda \varepsilon_{0}$. 
Remark. We conjecture that "most" homogeneous statistical solutions of the Navier-Stokes equations eventually "approach" a superposition of solutions of the type considered in Definition 1. This is the reason for the terminology introduced in this definition.

\section{The Correlation and the Energy Decay}

4.1. Let us recall that for a homogeneous (probability) measure satisfying $e(\mu)<\infty$, the correlation matrix $R(y)=\left[R_{j k}(y)\right]_{j, k=1}^{3}$ is defined by

$$
R_{j k}(y)=\int \frac{1}{|Q|_{Q}} \int_{Q} u_{j}(x+y) u_{k}(x) d x d \mu(u),
$$

where $j, k=1,2,3, y \in \mathbb{R}^{3}$ and $Q$ is any cube $(a, b)^{3}$. Obviously

$$
e(\mu)=\frac{1}{2} \operatorname{Tr} \mathrm{R}(0)=\frac{1}{2}\left(R_{11}(0)+R_{22}(0)+R_{33}(0)\right),
$$

and, if moreover $E(\mu)<\infty$, then $R_{j k}(j, k=1,2,3)$ is of class $C^{2}$ and

$$
E(\mu)=-\left.\Delta \operatorname{TrR}(y)\right|_{y=0}
$$

4.2. From now on until the end of this Sect. $4,\left(\mu^{v, \varepsilon}\right)_{v, \varepsilon>0}$ will be a fixed self-similar universal homogeneous statistical solution of the Navier-Stokes equations. We denote by $R(y ; v, \varepsilon)$ the correlation matrix of $\mu^{v, \varepsilon}$ (for all $v, \varepsilon>0$ ).

Proposition 2. Setting

$$
l=\left(\frac{\varepsilon}{v^{3}}\right)^{-1 / 4}
$$

we have

$$
R(y ; v, \varepsilon)=\varepsilon^{2 / 3} l^{2 / 3} R\left(\frac{y}{l} ; 1,1\right)
$$

for all $v, \varepsilon>0$ and $y \in \mathbb{R}^{3}$. Consequently

$$
e\left(\mu^{v, \varepsilon}\right)=\gamma(\varepsilon v)^{1 / 2}
$$

where

$$
\gamma=e\left(\mu^{1,1}\right)
$$

Proof. By Proposition 1, we have

$$
\begin{aligned}
& R\left(y ; \frac{\nu \xi}{\lambda}, \xi^{3} \lambda \varepsilon\right)=\left[\int \frac{1}{|Q|} \int_{Q} u_{j}(x+y) u_{k}(x) d x d \sigma_{\xi, \lambda}\left(\mu^{v, \varepsilon}(u)\right)\right]_{j, k}^{3} \\
& =\left[\xi^{2} \int \frac{1}{|Q|} \int_{Q} u_{j}(\lambda x+\lambda y) u_{k}(\lambda x) d x d \mu^{v, \varepsilon}(u)\right]_{j, k=1}^{3} \\
& =\xi^{2}\left[\int \frac{1}{|\lambda Q|} \int_{\lambda Q} u_{j}(x+\lambda y) u_{k}(x) d x d \mu^{v, \varepsilon}(u)\right]_{j, k=1}^{3} \\
& =\xi^{2} R(\lambda y ; v, \varepsilon)
\end{aligned}
$$


for all $y \in \mathbb{R}^{3}$. Replacing $y$ by $y / \lambda$ and choosing

$$
\xi=(v \varepsilon)^{-1 / 4}, \lambda=v^{3 / 4} \varepsilon^{-1 / 4} .
$$

we obtain (4.4). Taking $y=0$ in (4.4), by (4.1), we obtain readily (4.5).

Corollary. Let $\left(\mu^{v, \varepsilon(t)}\right)_{t \geqq 0}$ be any homogeneous statistical solution considered in Definition 1. Then for all $t \geqq 0$

$$
\varepsilon(t)=\varepsilon_{0}\left(1+\frac{t \varepsilon_{0}^{1 / 2}}{\gamma \nu^{1 / 2}}\right)^{-2}
$$

and consequently

$$
e\left(\mu^{v, \varepsilon(t)}\right)=e\left(\mu^{v, \varepsilon_{0}}\right)\left(1+\frac{t \varepsilon_{0}^{1 / 2}}{\gamma \nu^{1 / 2}}\right)^{-1}=e\left(\mu^{v, \varepsilon_{0}}\right)\left(1+\frac{t e\left(\mu^{v, \varepsilon_{0}}\right.}{\gamma^{2} v}\right)^{-1} .
$$

Proof. Obviously (4.8) is a direct corollary of (4.7) and (4.5). Also, because of (4.5), (3.8) and (3.10), we have

$$
\left.\frac{d(v \varepsilon(t))^{1 / 2} \gamma}{d t}+\varepsilon(t)=0 \text { (for } t \geqq 0\right) \text { and } \varepsilon(0)=\varepsilon_{0},
$$

from where by an elementary computation we obtain (4.8).

Remark. (by O. P. Manley). Formula (4.9) implies

$$
t e\left(\mu^{v, \varepsilon(t)}\right) \rightarrow v \gamma^{2} \text { for } t \rightarrow \infty,
$$

which represents a universal law of decay (i.e. a law independent of the initial date $\left.\mu^{v, \varepsilon_{0}}\right)$.

\section{Connection with Equations (2)}

5.1 In this paragraph we establish the connection between the self-similar universal homogeneous statistical solution of the Navier-Stokes equations and the stationary homogeneous statistical solutions of Eqs. (2). Namely we have the following

Theorem. $\left(\mu^{v, \varepsilon}\right)_{v, \varepsilon>0}$ is a self-similar universal homogeneous statistical solution of the Navier-Stokes equations if and only if it is of the form (see (4.3.))

$$
\mu^{v, \varepsilon}=\sigma_{\gamma^{-1 / 2} \varepsilon^{1 / 4} v^{1 / 4}, \gamma^{-1 / 2} \varepsilon^{1 / 4} v^{-3 / 4}}(\mu)(\text { for all } \nu, \varepsilon>0)
$$

for some stationary homogeneous statistical solution $\mu$ of Eqs. (2) satisfying

$$
e(\mu)=E(\mu)\left(=\gamma^{2}\right) \text {. }
$$

The next two Sects, 5.2 and 5.3, will be devoted to the proof of this Theorem. 5.2. Let $\left(\mu^{v, \varepsilon}\right)_{v, \varepsilon>0}$ be a self-similar universal homogeneous statistical solution of the Navier-Stokes equations. We set

$$
\mu=\mu^{1, \gamma^{2}}
$$

where $\gamma$ was introduced in (4.6). Then (5.1) is satisfied by virtue of Proposition 1, 
while (5.2) follows directly from (3.8) and (4.5). So it remains to prove that $\mu^{1, \gamma^{2}}$ is a stationary homogeneous solution of Eqs. (2). To this aim, set

$$
\tau=\gamma v^{1 / 2} \varepsilon^{-1 / 2}
$$

and observe that

$$
\mu^{v, \varepsilon}=\sigma_{(v / \tau)^{1 / 2},(v \tau)^{-1 / 2}}(\mu),
$$

so that if $\left(\mu^{v, \varepsilon(t)}\right)_{t \geqq 0}$ is the homogeneous statistical solution considered in Sect. 4 , then for $\tau(t)=\gamma v^{1 / 2} \varepsilon(t)^{-1 / 2}$, we have

$$
\frac{d \tau(t)}{d t}=1
$$

(see (4.10)). Therefore (giving to $\tau(t)$ the role of $t$ !)

$$
\left(\sigma_{(v / t)^{1 / 2},(v t)^{-1 / 2}}(\mu)\right)_{t \geqq 0}
$$

will satisfy Eq. (2.3) for any $\Phi \in \mathscr{T}$. But

$$
\begin{aligned}
& \frac{d}{d t} \int \Phi d \sigma_{(v / t)^{1 / 2},(v t)^{-1 / 2}}(\mu)=\frac{d}{d t} \int \Phi\left(\sigma_{(v / t)^{1 / 2},(v t)^{-1 / 2}} u d \mu(u)\right. \\
& \quad=-\frac{1}{2 t} \int\left(\sigma_{(v / t)^{1 / 2},(v t)^{-1 / 2}} u+(x \cdot \nabla) \sigma_{(v / t)^{1 / 2},(v t)^{-1 / 2}} u,\right. \\
& \left.\Phi^{\prime}\left(\sigma_{(v / t)^{1 / 2},(v t)^{-1 / 2}} u\right)\right) d \mu(u)=-\frac{1}{2 t} \int\left(u+(x \cdot \nabla) u, \Phi^{\prime}(u)\right) d \sigma_{(v / t)^{1 / 2},(v t)^{-1 / 2}}(\mu)(u),
\end{aligned}
$$

so that from (2.3) taking $v=1, t=1$ we obtain Eq. (2.7).

5.3. Conversely, let us assume that $\mu$ is a stationary homogeneous statistical solution of Eqs. (2) such that $e(\mu)=E(\mu)$. We take $\gamma^{2}=e(\mu)$ and define the family $\left(\mu^{v, \varepsilon}\right)_{v, \varepsilon>0}$ by (5.1). We shall show that this family is indeed a self-similar universal homogeneous statistical solution of the Navier-Stokes equations.

To this end, first we note that, by (3.7), (3.8) is satisfied. Also, using (3.4) and (5.7) we obtain (for $v, \varepsilon>0$ )

$$
\begin{aligned}
\tau \int & {\left[v\left(\left(u, \Phi^{\prime}(u)\right)\right)+\left((u \cdot \nabla) u, \Phi^{\prime}(u)\right)\right] d \sigma_{(v / \tau)^{1 / 2},(v \tau)^{-1 / 2}}(\mu)(u) } \\
= & \int\left[\left(\left(u, \nabla_{u}\left[\Phi\left(\sigma_{(v / \tau)^{1 / 2},(v \tau)^{-1 / 2}} u\right)\right]\right)\right)\right. \\
& \left.\left.+\left((u \cdot \nabla) u, \nabla_{u}\left[\Phi\left(\sigma_{(v / \tau)^{1 / 2},(v \tau)^{-1 / 2}} u\right)\right]\right)\right)\right] d \mu(u) \\
= & \left.\frac{1}{2} \int\left(u+(x \cdot \nabla) u, \nabla_{u}\left[\Phi\left(\sigma_{(v / \tau)^{1 / 2},(v \tau)^{-1 / 2}} u\right)\right]\right)\right) d \mu(u) \\
= & \frac{1}{2} \int\left(\sigma_{(v / \tau)^{1 / 2},(v \tau)^{-1 / 2}}(u+(x \cdot \nabla) u), \Phi^{\prime}\left(\sigma_{(v / \tau)^{1 / 2},(v \tau)^{-1 / 2}} u\right)\right) d \mu(u) \\
= & \frac{1}{2} \int\left(u+(x \cdot \nabla) u, \Phi^{\prime}(u)\right) d \sigma_{(v / \tau)^{1 / 2},(v \tau)^{-1 / 2}}(\mu)(u) \\
= & -\tau \frac{d}{d t} \int \Phi d \sigma_{(v / \tau)^{1 / 2},(v \tau)^{-1 / 2}}(\mu) .
\end{aligned}
$$

For $v, \varepsilon_{0}>0$ fixed, we define

$$
t=\tau-\tau_{0}, \text { where } \tau_{0}=\gamma \nu^{1 / 2} \varepsilon_{0}^{-1 / 2}
$$


and $\tau \geqq \tau_{0}$. Then if $\varepsilon(t)$ is given by formula (4.8), we have

$$
\tau=\gamma v^{1 / 2} \varepsilon(t)^{-1 / 2}
$$

and therefore

$$
\mu^{v, \varepsilon(t)}=\sigma_{(v / \tau)^{1 / 2},(v \tau)^{-1 / 2}}(\mu) \text { for all } \tau \geqq \tau_{0} .
$$

By (5.8) it follows that $\left(\mu^{v, \varepsilon(t)}\right)_{t \geqq 0}$ satisfies (2.3) for any $\Phi \in \mathscr{T}$. Moreover since, by (3.7) and (5.9)

$$
e\left(\mu^{v, \varepsilon(t)}\right)=e\left(\sigma_{(v / \tau)^{1 / 2},(v \tau)^{-1 / 2}}(\mu)\right)=\frac{\nu}{\tau} e(\mu)=\gamma^{2} v / \tau=\gamma(v \varepsilon(t))^{1 / 2},
$$

it follows that $\mu_{t}=\mu^{v, \varepsilon(t)}(t \geqq 0)$ satisfies also (3.10). Since this family $\left(\mu_{t}\right)_{t \geqq 0}$ obviously satisfies (2.1), we conclude that $\left(\mu^{v, \varepsilon(t)}\right)_{t \geqq 0}$ is the homogeneous statistical solution which satisfies the conditions of Definition 1. So $\left(\mu^{v, \varepsilon}\right)_{v, \varepsilon>0}$ is a universal homogeneous statistical solution of the Navier-Stokes equations. Since, by its very definition, this solution satisfies (3.11), the proof is finished.

5.4. We supplement the preceding theorem with the following remark. Let $\left(\mu^{v, \varepsilon}\right)_{v, \varepsilon>0}$ be a self-similar universal homogeneous statistical solution of the NavierStokes equations and let $\mu=\mu^{1, \gamma^{2}}$ (where $\gamma=e\left(\mu^{1,1}\right)$ ) be the stationary homogeneous statistical solution of Eqs. (2) considered in the preceding theorem. Let moreover $R(y)$ be the correlation matrix of $\mu$. Then

$$
R(y ; v, \varepsilon)=\gamma^{-1} \varepsilon^{2 / 3} l^{2 / 3} R\left(y \gamma^{-1 / 2} l^{-1}\right)
$$

for all $v, \varepsilon>0$ and $y \in \mathbb{R}^{3}$; here $l$ is given by (4.3), i.e. $l=\left(\varepsilon / v^{3}\right)^{-1 / 4}$. Also

$$
\mu^{v, \varepsilon}=\sigma_{\gamma^{-1 / 2} v l^{-1}, \gamma^{-1 / 2 l^{-1}}}(\mu) \text { (for all } v, \varepsilon>0 \text { ). }
$$

Indeed, (5.11) is obvious and (5.10) follows at once from (4.4) and its version for $R\left(y ; 1, \gamma^{2}\right)$.

\section{Supplementary Properties}

6.1. As shown in [10], the proof of the existence theorem of Sect. 1, given in [9], implies also the basic property that

$$
\int u d \mu_{t}=\int u d \mu_{0} \text { for all } t \geqq 0,
$$

i.e.

$$
\int \frac{1}{|Q|} \int_{Q} u d x d \mu_{t}(u)=\int \frac{1}{|Q|} \int_{Q} u d x d \mu_{0}(u) \text { for all } t \geqq 0,
$$

where, as in Sect. $1, Q$ is any bounded measurable subset of $\mathbb{R}^{3}$ such that $|Q|>0$.

Proposition 3. The homogeneous statistical solution of the Navier-Stokes equations occurring in Definition I enjoys the property (6.1) if and only if

$$
\int u d \mu^{v, \varepsilon}=0 \text { for all } \nu, \varepsilon>0 .
$$


If $\mu$ is the stationary homogeneous statistical solution of Eqs. (2) considered in the theorem of Sect. 5, then (6.3) is equivalent to

$$
\int u d \mu=0 .
$$

Proof. If (6.3) holds, so does (6.4) since $\mu=\mu^{1, \gamma^{2}}$. Also, by (5.1) and the homogeneity of $\mu$,

$$
\begin{aligned}
& \int \frac{1}{|Q|} \int_{Q} u d x d \mu^{v, \varepsilon}=\int \frac{1}{|Q|} \int_{Q} \sigma_{\xi, \lambda} u d x d \mu \\
& \quad=\xi \int \frac{1}{|\lambda Q|} \int_{\lambda Q} u(x) d x d \mu=\xi \int \frac{1}{|Q|} \int_{Q} \mu(u) d x d \mu
\end{aligned}
$$

for any $Q$ as in (6.2), where

$$
\xi=\gamma^{-1 / 2} \varepsilon^{1 / 4} v^{1 / 4}, \lambda=\gamma^{-1 / 2} \varepsilon^{1 / 4} v^{-3 / 4} .
$$

Thus, conversely, (6.4) implies (6.3). Finally if for some $v, \varepsilon_{0}>0,\left(\mu^{v, \varepsilon(t)}\right)_{0 \leqq t}<\infty$ is the homogeneous statistical solution of the Navier-Stokes equations considered in Definition 1, then by (6.5), (6.6),

$$
\int u d \mu^{v, \varepsilon(t)}=\gamma^{-1 / 2} \varepsilon(t)^{1 / 4} v^{1 / 4} \int u d \mu(u) \text { for all } t \geqq 0 .
$$

By virtue of (4.8) this quantity is independent of $t$ if and only if (6.4) holds. 6.2. Concerning the relation (5.2) we have the following

Proposition 4. Let $\mu$ be a stationary homogeneous statistical solution of Eqs. (2) satisfying the following regularity property

$$
\int|\Delta u|_{Q}^{2} d \mu(u)<\infty
$$

for some cube $Q$ (thus also for any bounded measurable subset $Q$ of $\mathbb{R}^{n},|Q|>0$ ), and the following growth condition

$$
\int \frac{1}{|Q|} \int_{Q}|u(x)|^{4} d x d \mu(u)<\infty .
$$

Then $\mu$ satisfies Eqs. (5.2) and (6.4).

Proof. We consider first a $\Phi \in \mathscr{T}$ of the form

$$
\Phi(u)=\varphi\left(\sum_{j=1}^{k}\left(u, g_{j}\right)^{2}\right)
$$

where $g_{1}, g_{2}, \ldots, g_{k} \in V_{c}$ and $\varphi$ is a real $\mathscr{C}^{1}$ function on $\mathbb{R}$ bounded together with its derivative, for which (2.7) becomes

$$
\int \varphi^{\prime}\left(\sum_{j=1}^{k}\left(u, g_{j}\right)^{2}\right)+\sum_{j=1}^{k}\left(-\frac{1}{2} u-\frac{1}{2}(x \cdot \nabla) u+(u \cdot \nabla) u-\Delta u, g_{j}\right)\left(u, g_{j}\right) d \mu(u)=0
$$

We can easily choose a sequence $\left\{\varphi_{j}\right\}_{j=1}^{\infty}$ of functions $\varphi$ as in (6.9) such that

$$
\sup _{j, x} \varphi_{j}(\rho)<\infty, \varphi_{j}(\rho) \rightarrow 1 \text { for all } \rho \in \mathbb{R}
$$


Then by virtue of (2.6), (6.7) and (6.8), we obtain from (6.10) (written for $\varphi=\varphi_{j}$ ) the following relation

$$
\int \sum_{j=1}^{k}\left(-\frac{1}{2} u-\frac{1}{2}(x \cdot \nabla) u+(u \cdot \nabla) u-\Delta u, g_{j}\right)\left(u, g_{j}\right) d \mu(u)=0 .
$$

Moreover we can choose a sequence $\left\{g_{j}\right\}_{j=1}^{\infty} \subset V_{c}$ (with sup $g_{j} \subset Q, \forall j$ ) which should be an orthonormal basis in $L^{2}(Q)$. Then again by virtue of $(2.6),(6.7)$ and (6.8), we obtain from (6.10) (by letting $k \rightarrow \infty$ )

$$
\iint_{Q}\left(-\frac{1}{2} u-\frac{1}{2}(x \cdot \nabla) u-\Delta u\right) \cdot u d x d \mu(u)=0 .
$$

In (6.12) we used that fact that

$$
\iint_{Q}[(u \cdot \nabla) u] \cdot u d \mu(u) d x=\sum_{j=1}^{3} \int_{Q} D_{j}\left|\int u_{j} \frac{|u(x)|^{2}}{2} d \mu(u)\right| d x=0,
$$

because of the homogeneity of $\mu$. Thus dividing by $|Q|$ we obtain (see Sect. 4.1)

$$
\begin{aligned}
E(\mu)-e(\mu) & =\frac{1}{2} \int \frac{1}{|Q|} \int_{Q}(x \cdot \nabla) u \cdot u d x d \mu(u) \\
& =\frac{1}{2} \int \frac{1}{|Q|} \int_{Q}(x \cdot \nabla) \frac{1}{2}|u(x)|^{2} d x d \mu(u)=\frac{1}{2} \frac{1}{|Q|} \int(x \cdot \nabla) e(\mu) d x=0 .
\end{aligned}
$$

Also if we consider a $\Phi \in \mathscr{T}$ of the form

$$
\Phi(u)=\varphi\left(\sum_{j=1}^{k}\left(u, g_{j}\right)\left(g_{j}, g\right)\right)
$$

where $g, g_{1}, \ldots, g_{k} \in V_{c}$ and $\varphi$ is as in (6.9), (2.7) becomes

$$
\begin{aligned}
& \int \varphi^{\prime}\left(\sum_{j=1}^{k}\left(u, g_{j}\right)\left(g_{j}, g\right)\right) \sum_{j=1}^{k}\left(-\frac{1}{2} u-\frac{1}{2}(x \cdot \nabla) u-\Delta u\right. \\
& \left.\quad+(u \cdot \nabla) u, g_{j}\right)\left(g_{j}, g\right) d \mu(u)=0 .
\end{aligned}
$$

Proceeding with (6.13) as with (6.10) we obtain, instead of (6.12), the relation

$$
\iint_{Q}\left(-\frac{1}{2} u-\frac{1}{2}(x \cdot \nabla) u-\Delta u+(u \cdot \nabla) u\right) \cdot g d x d \mu(u)=0,
$$

from which we infer

$$
\begin{aligned}
\frac{1}{2} \int u d \mu \cdot \int_{Q} g d x= & -\frac{1}{2} \int_{Q}(x \cdot \nabla) \cdot \int u d \mu(u) \cdot g d x \\
& -\int_{Q} \Delta \int u d \mu(u) \cdot g d x+\iint_{Q}(u \cdot \nabla) u \cdot g d x d \mu(u) \\
= & \iint_{Q}(u \cdot \nabla) u \cdot g d x d \mu(u),
\end{aligned}
$$

since $\int u d \mu(u)$ is independent of $x$. But

$$
\iint_{Q}(u \cdot \nabla) u \cdot g d x d \mu(u)=\sum_{j, k=1}^{3} \iint_{Q} u_{j} D_{j} u_{k} \cdot g_{k} d x d \mu(u)
$$




$$
=\sum_{j, k=1}^{3} \iint_{Q} D_{j}\left(u_{j} u_{k}\right) \cdot g_{k} d x d \mu(u)=\sum_{j, k=1}^{3} \int_{Q}\left(D_{j}\left(R_{j k}(0)\right)\right) g_{k} d x=0,
$$

so that (6.14) yields

$$
\int u d \mu \cdot \int_{Q} g d x=0
$$

for all $g \in V_{c}$. Taking $g=\operatorname{curl} h$, where $h \in C_{0}^{\infty}\left(\mathbb{R}^{3}\right)^{3}$ is arbitrary, we easily verify that

$$
\left\{\int_{Q} g d x ; g \in V_{c}\right\}=\mathbb{R}^{3}
$$

so that (6.15) implies (6.1).

Remark. The preceding proof shows that if instead of our Eqs. (2) one considers the time dependent form of the Leray equations (3), then one obtains $E(\mu)+e(\mu)=0$, instead of $E(\mu)-e(\mu)=0$. Obviously the first relation implies that $\mu$ is carried by the null flow, i.e. $\mu$ is a trivial solution. Thus we can conclude that the Leray equations (3) have no nontrivial stationary homogeneous statistical solutions satisfying the regularity property (6.7) and the growth condition (6.8).

\section{Remarks on the Energy Spectrum}

7.1. Let $\mu$ be a homogeneous (probability) measure on $H_{\text {loc }}$ such that its correlation matrix $R(y)$ exists and $\operatorname{Tr} R(y)$ is the Fourier transform

$$
\left.\operatorname{Tr} R(y)=\int_{\mathbb{R}^{3}} e^{i k \cdot y} Q(k) d k \text { (for all } y \in \mathbb{R}^{3}\right)
$$

of a function $Q \in L^{1}\left(\mathbb{R}^{3}\right)$. Since $\operatorname{Tr} R(y)$ is the Fourier transform of a positive measure (see $|3|$ ), $Q \geqq 0$. The energy spectrum of $\mu$ is the function defined for all $\kappa>0$, by

$$
S(\kappa)=\int_{|k|=\kappa} Q(k) d \Sigma(k)
$$

where $d \Sigma(k)$ denotes the area element of the sphere (in $\mathbb{R}^{3}$ ) of radius $\kappa$. Obviously

$$
e(\mu)=\frac{1}{2} \int_{0}^{\infty} S(\kappa) d \kappa
$$

If moreover $E(\mu)<\infty$, then (using (4.2)) it is easy to check that

$$
E(\mu)=\int_{0}^{\infty} \kappa^{2} S(\kappa) d \kappa
$$

7.2. Proposition. Let $\mu$ be a stationary homogeneous statistical solution of Eqs. (2) satisfying (5.2.) and let $\left(\mu^{v, \varepsilon}\right)_{v, \varepsilon>0}$ be the self similar universal homogeneous statistical solutions of the Navier-Stokes equations corresponding to $\mu$ according to the theorem in Sect. 5.1. If $\mu$ has an energy spectrum $S(\kappa)$, then for all $v, \varepsilon>0, \mu^{v, \varepsilon}$ has an energy 
spectrum $S(\kappa ; v, \varepsilon)$ given by

$$
S(\kappa ; v, \varepsilon)=\gamma^{-1 / 2} \varepsilon^{2 / 3} \kappa_{d}^{-5 / 3} S\left(\gamma^{1 / 2} \kappa / \kappa_{d}\right),
$$

where

$$
\kappa_{d}=\left(\frac{\varepsilon}{v^{3}}\right)^{1 / 4} .
$$

Proof. By virtue of the remark made in Sect. 5.4, we have

$$
\begin{aligned}
\operatorname{Tr} R(y ; v, \varepsilon) & =\gamma^{-1} \varepsilon^{2 / 3} \kappa_{d}^{-2 / 3} \operatorname{Tr} R\left(\gamma^{-1 / 2} \kappa_{d} y\right) \\
& =\gamma^{-1} \varepsilon^{2 / 3} \kappa_{d}^{-2 / 3} \int_{\mathbb{R}^{3}} e^{i \gamma^{-1 / 2} \kappa_{d} y \cdot k} Q(k) d k \\
& =\gamma^{1 / 2} \varepsilon^{2 / 3} \kappa_{d}^{-11 / 3} \int_{\mathbb{R}^{3}} e^{i y \cdot k} Q\left(\gamma^{1 / 2} k / \kappa_{d}\right) d k
\end{aligned}
$$

hence, by (7.2)

$$
\begin{aligned}
S(\kappa ; \nu, \varepsilon) & =\int_{|k|=\kappa} \gamma^{1 / 2} \varepsilon^{2 / 3} \kappa_{d}^{-11 / 3} Q\left(\gamma^{1 / 2} k / \kappa_{d}\right) d \Sigma(k) \\
& =\gamma^{-1 / 2} \varepsilon^{2 / 3} \kappa_{d}^{-5 / 3} \int_{|k|=\frac{\gamma^{12} \kappa}{\kappa_{d}}} Q(k) d \Sigma(k) .
\end{aligned}
$$

i.e. (7.5).

7.3. Remark. If we define

$$
F(\kappa)=\gamma^{-1 / 2} \kappa^{5 / 3} S\left(\gamma^{1 / 2} \kappa\right)(\text { for } \kappa>0),
$$

then (7.5) takes the form

$$
S(\kappa ; v, \varepsilon)=\varepsilon^{2 / 3} \kappa^{-5 / 3} F\left(\kappa / \kappa_{d}\right)(\text { for } \kappa>0) \text {. }
$$

Comparing (7.8) with [15] Sect. 3.1, we see that this is the usual form of the energy spectrum of turbulence. However our formula (7.7) gives a new mathematical interpretation of the function $F(\kappa)$. In particular, since

$$
\frac{1}{2} \int_{0}^{\infty} S(\kappa) d \kappa=e(\mu)=\gamma^{2}=E(\mu)=\int^{\infty} \kappa^{2} S(\kappa) d \kappa,
$$

the function $F(\kappa)$ satisfies the following two conditions

$$
\frac{1}{2} \int_{0}^{\infty} F(\kappa) \kappa^{-5 / 3} d \kappa=\gamma, \int_{0}^{\infty} F(\kappa) \kappa^{1 / 3} d \kappa=1 .
$$

\section{Connection to Conventional Turbulence Theory}

We give now some comments of physical nature, some of those in Sect. 8.3 being purely speculative ones.

8.1. Let $\left(\mu^{v, \varepsilon}\right)_{v, \varepsilon>0}$ be a fixed self-similar universal homogeneous statistical solution 
of the Navier-Stokes equations satisfying (6.1). Here $\mu^{v, \varepsilon}$ concerns an incompressible fluid in which:

$$
\text { kinematic viscosity }=v \mathrm{~cm}^{2} / \mathrm{s},
$$

and (by virtue of (4.5)),

$$
\text { mean energy per } \mathrm{g}=\gamma(v \varepsilon)^{1 / 2} \mathrm{~cm}^{2} / \mathrm{s}^{2},
$$

(where gr denotes the unit of mass $=$ gram) and

$$
\text { mean energy dissipation per } \mathrm{g}=\varepsilon \mathrm{cm}^{2} / \mathrm{s}^{3} \text {. }
$$

Obviously the mean energy dissipation time should be

$$
\frac{\text { mean energy per gr }}{\text { mean energy dissipation per } \mathrm{g}} \mathrm{s}
$$

so that by $(8.2),(8.3)$, we obtain

$$
\text { mean energy dissipation time }=\gamma(\nu / \varepsilon)^{1 / 2} \mathrm{~s} .
$$

Taking into account (4.9) we deduce that the mean energy dissipation time is equal to the half life time of the mean energy per $\mathrm{g}$.

8.2. Since $\left(\mu^{v, \varepsilon}\right)_{v, \varepsilon>0}$ satisfies (6.1), Proposition 3 and (5.1) imply

$$
\int u d \mu^{v, \varepsilon}=0 \quad(\text { for all } v, \varepsilon>0) .
$$

Therefore we have that the mean velocity is

$$
(2 \text { mean energy per } \mathrm{g})^{1 / 2} \text {, }
$$

that is (by (8.2))

$$
\text { mean velocity }=(2 \gamma)^{1 / 2}(v \varepsilon)^{1 / 4} \mathrm{~cm} / \mathrm{s} .
$$

For the natural mean length, given by mean velocity, mean energy dissipation time, we have (see (8.8) and (8.4))

$$
\begin{aligned}
\text { mean length } & =(2 \gamma)^{1 / 2}(v \varepsilon)^{1 / 4} \gamma(v / \varepsilon)^{1 / 2} \mathrm{~cm} \\
& =2^{1 / 2} \gamma^{3 / 2}\left(v^{3} / \varepsilon\right)^{1 / 4} \mathrm{~cm} .
\end{aligned}
$$

It is obvious that by introducing Kolmogorov's

$$
\text { dissipation length: }\left(v^{3} / \varepsilon\right)^{1 / 4} \mathrm{~cm} \text {, }
$$

we can write

$$
\text { mean length }=2^{1 / 2} \gamma^{3 / 2} \text {. dissipation length. }
$$

On the other hand the Reynolds number of the flow is given by

$$
\begin{aligned}
R & =\frac{\text { mean velocity } \cdot \text { mean length }}{v} \\
& =\frac{(2 \gamma)^{1 / 2}(v \varepsilon)^{1 / 4} 2^{1 / 2} \gamma^{3 / 2}\left(v^{3} / \varepsilon\right)^{1 / 4}}{v}=2 \gamma^{2} .
\end{aligned}
$$


Thus the Reynolds number is time invariant, and (8.9) becomes

$$
\frac{\text { mean length }}{\text { dissipation length }}=2^{1 / 4} R^{3 / 4} \text {. }
$$

It follows that Landau's number of degrees of freedom, which is the cube of the left hand side of $(8.11)$, is $2^{3 / 4} R^{9 / 4}$. For a reader familiar with [1, 15, 13, Sects. 31 and 32], all the relations are in agreement with the corresponding ones in the conventional turbulence theory. However our approach is more rigorous than the usual empirical one, based on purely dimensional arguments, i.e. the approach in the conventional turbulence theory.

In particular, let us notice that according to (4.8) and (8.10), $\varepsilon=\varepsilon\left(\mu^{v, \varepsilon(t)}\right)$ satisfies the differential equation

$$
\frac{d \varepsilon}{d t}=-\frac{(2 \varepsilon)^{3 / 2}}{(R v)^{1 / 2}} \text { for } t>0 .
$$

Therefore for an approximative equilibrium (i.e. $\varepsilon \sim$ const $\sim 1 \mathrm{~cm}^{2} / \mathrm{s}^{3}$ as long as $t$ $\sim 1 \mathrm{~s}$.), we must have $R \gg 1$, since for most interesting fluids $v<1 \mathrm{~cm}^{2} / \mathrm{s}$.

8.3. We shall now discuss briefly the consistence of our formula (7.5-9) and (8.7-11) with Kolmogorov's energy spectrum theory. The basic conclusions of this theory can be summarized as follows (see [12] Sects. 3.1-3, Sect. 2.9 or [4], Chap. II, Sect. 4): Set $\kappa_{L} \sim R^{-3 / 4} \kappa_{d}$, where $\kappa_{d}=\left(\varepsilon / v^{3}\right)^{1 / 4}$ is the reciprocal of the dissipation length (8.8) and the Reynolds number $R$ is assumed to be $\gg 1$. Then

$$
\begin{gathered}
\int_{\kappa}^{\infty} S(\kappa ; v, \varepsilon) d \kappa \ll \int_{0}^{\infty} S(\kappa ; \nu, \varepsilon) d \kappa, \\
\int_{0}^{\kappa} \kappa^{2} S(\kappa ; v, \varepsilon) d \kappa \ll \int_{0}^{\infty} \kappa^{2} S(\kappa ; \nu, \varepsilon) d \kappa,
\end{gathered}
$$

and, for $\kappa$ in the initial range (i.e. $\kappa_{L}<\kappa<\kappa_{d}$ ), the energy spectrum $S(\kappa ; v, \varepsilon)$ is approximately equal to

$$
C \varepsilon^{2 / 3} \kappa^{-5 / 3}\left(\kappa / \kappa_{L}\right)^{-h}
$$

where the term $\left(\kappa / \kappa_{L}\right)^{-h}$ accounts for the intermittency phenomena, and $h, C>0$ are dimensionless constants. Experiments suggest that $h \ll 1$ and $C \sim 1$.

We start by proving that in our case, the relations $(8.12-13)$ are satisfied for $R \gg 1$. Indeed by virtue of (7.5), (7.7-8), (7.3), (4.5) and (8.10) we have

$$
\begin{aligned}
& \int_{\kappa_{d}}^{\infty} S(\kappa ; \nu, \varepsilon) d \kappa=\varepsilon^{2 / 3} \int_{\kappa_{d}}^{\infty} \kappa^{-5 / 3} F\left(\kappa / \kappa_{d}\right) d \kappa \\
& =\varepsilon^{2 / 3} \kappa_{d}^{-2 / 3} \int_{1}^{\infty} \kappa^{-5 / 3} F(\kappa) d \kappa \leqq \varepsilon^{2 / 3} \kappa_{d}^{-2 / 3} \int_{1}^{\infty} \kappa^{1 / 3} F(\kappa) d \kappa \\
& \leqq \varepsilon^{2 / 3} \kappa_{d}^{-2 / 3}=(\varepsilon v)^{1 / 2}=\left(\frac{2}{R}\right)^{1 / 2} e\left(\mu^{v, \varepsilon}\right) \\
& =\frac{1}{(2 R)^{1 / 2}} \int_{0}^{\infty} S(\kappa ; v, \varepsilon) d \kappa,
\end{aligned}
$$


i.e.

$$
\int_{\kappa_{d}}^{\infty} S(\kappa ; \nu, \varepsilon) d \kappa \leqq \frac{1}{(2 R)^{1 / 2}} \int_{0}^{\infty} S(\kappa ; \nu, \varepsilon) d \kappa .
$$

Concerning relation (8.13) we first notice that by virtue of (8.11) an acceptable value for $\kappa_{L}$ is

$$
\kappa_{L}=\frac{1}{\text { mean length }}=2^{-1 / 4} R^{-3 / 4} \kappa_{d},
$$

and that, by virtue of (7.5), (7.7-8), (8.10), (7.4) and (3.12), we have

$$
\begin{aligned}
& \int_{0}^{\kappa} \kappa^{2} S(\kappa ; v, \varepsilon) d \kappa=\varepsilon^{2 / 3} \int_{0}^{\kappa} \kappa^{1 / 3} F\left(\kappa / \kappa_{d}\right) d \kappa \\
& =\varepsilon^{2 / 3} \kappa_{L}^{2} \int_{0}^{\kappa} \kappa^{-5 / 3} F\left(\kappa / \kappa_{d}\right) d \kappa=\varepsilon^{2 / 5} \kappa_{L}^{2} \kappa_{L}^{-2 / 3} \int_{0}^{2-1 / 4 R^{-3 / 4}} \kappa^{-5 / 3} F(\kappa) d \kappa \\
& \leqq \varepsilon^{2 / 3} \kappa_{L}^{2} \kappa_{d}^{-2 / 3} 2\left(\frac{R}{2}\right)^{1 / 2}=\varepsilon^{2 / 3} \kappa_{d}^{4 / 3} \frac{1}{R}=\frac{\varepsilon}{v} \frac{1}{R} \\
& =\frac{1}{R} \int_{0}^{\infty} \kappa^{2} S(\kappa ; v, \varepsilon) d \kappa,
\end{aligned}
$$

i.e.

$$
\int_{0}^{\kappa_{L}} \kappa^{2} S(\kappa ; v, \varepsilon) d \kappa \leqq \frac{1}{R} \int_{0}^{\infty} \kappa^{2} S(\kappa ; v, \varepsilon) d \kappa .
$$

Clearly, (8.14) and (8.17) show that (8.12-13) hold if $R \gg 1$.

In a speculative mode we can assume that for $R \gg 1$ we have the following stronger version of $(8.12-13)$

$$
\begin{aligned}
& \int_{\kappa_{L}}^{\kappa_{d}} S(\kappa ; v, \varepsilon) d \kappa \sim \int_{0}^{\infty} S(\kappa ; v, \varepsilon) d \kappa \quad\left(=(2 v \varepsilon R)^{1 / 2}\right), \\
& \int_{\kappa_{L}}^{\kappa_{d}} \kappa^{2} S(\kappa ; v, \varepsilon) d \kappa \sim \int_{0}^{\infty} \kappa^{2} S(\kappa ; v, \varepsilon) d \kappa \quad\left(=\frac{\varepsilon}{v}\right),
\end{aligned}
$$

and that, indeed $S(\kappa ; v, \varepsilon)$ has the form (8.14) for $\kappa_{L}<\kappa<\kappa_{d}$ with a constant $C$ depending only on the Reynolds number $R$. Experimental data as well as theoretical deduction suggest that $h \sim 4 / 30 .^{3}$ Then (8:18) yields

$$
\begin{aligned}
C(R) \frac{\left(2^{1 / 3} \varepsilon v R\right)^{1 / 2}}{8} & \sim \frac{C(R) \varepsilon^{2 / 3}}{\left(\frac{2}{3}+h\right) \kappa_{d}^{2 / 3}}\left(2^{1 / 6} R^{1 / 2}-\frac{1}{2^{1 / 4} R^{3 / 4}}\right) \\
& =\int_{\kappa_{L}}^{\kappa_{d}} S(\kappa ; \nu, \varepsilon) d \kappa \sim(2 v \varepsilon R)^{1 / 2},
\end{aligned}
$$

hence

$$
C(R) \sim .8 \times 2^{1 / 3} \sim 1 .
$$


On the other hand, (8.19) yields

$$
\begin{aligned}
\frac{C(R)}{1.2} \frac{\varepsilon}{\nu}\left(\frac{1}{2^{1 / 3} R}\right)^{.1} & \sim \frac{C(R) \varepsilon^{2 / 3}}{\left(\frac{3}{4}-h\right) \kappa_{d}^{4 / 3}}\left|\left(\frac{\kappa_{L}}{\kappa_{d}}\right)^{h}-\frac{1}{2^{1 / 3} R}\right| \\
& =\int_{\kappa_{L}}^{\kappa_{d}} \kappa^{2} S(\kappa ; \nu, \varepsilon) d \kappa \sim \frac{\varepsilon}{v}
\end{aligned}
$$

so that

$$
C(R) \sim 1.2 \times\left(2^{1 / 3} R\right)^{\cdot 1} .
$$

Obviously (8.21) shows that $C(R)$ seemingly depends on $R$ but that this dependence is so mild that (8.20) and (8.21) are actually compatible for $10^{-4} \leqq R \leqq 10^{4}$. So $(8.15)$ might be an acceptable approximation to the energy spectra occurring in our approach.

\section{References}

1. Batchelor, G. K.: The theory of homogeneous turbulence. Cambridge: Cambridge University Press 1967

2. Bensoussan, A., Temam, R.: Equations stochastiques de type Navier-Stokes. J. Funct. Anal. 13, 195222 (1973)

3. Cafarelli, L., Kohn, R., Nirenberg, L.: Partial regularity of suitable weak solutions of the NavierStokes equations. Preprint (to appear)

4. Chorin, A. J.: Lectures on Turbulence. Boston: Publish or Perish Inc. 1975

5. Foias, C.: Ergodic problems in functional spaces related to Navier-Stokes equations. Proc. Intern. Conf. Function. Anal. and related topics, Tokyo (1969) pp. 290-304

6. Foias, C.: Statistical study of the Navier-Stokes equations. I. Rend. Sem. Mat. Univ. Padova 48, 219348 (1972) II., idem, 49, 9-123 (1973)

7. Foias, C., Manley, O. P., Temam, R.: A new representation of Navier-Stokes equations governing self-similar homogeneous turbulence. (in preparation)

8. Foias, C., Temam, R.: Some analytic and geometric properties of the solutions of the Navier-Stokes equations. J. Math. Pures Appl. 58, 339-368 (1979)

9. Foias, C., Temam, R.: Homogeneous statistical solutions of the Navier-Stokes equations. Indiana Univ. Math. J. 29, 913-957 (1980)

10. Foias, C., Temam, R.: Homogeneous statistical solutions of the Navier-Stokes equations, II. (in preparation)

11. Hopf, E. : Statistical hydrodynamic and functional calculus. J. Rat. Mech. Anal. 16, 87 - 123 (1948)

12. Ladysenskaya, O. A., Vershik, A. M.: Sur l'évolution des mesures déterminées par les équations de Navier-Stokes et la résolution du probleme de Cauchy pour l'équation statistique de Hopf. Ann. Scuola Norm. Sup. Pisa; S. IV 4, 209-230 (1977)

13. Landau, L. D., Lifshitz, E. M.: Fluid mechanics. New York: Pergamon 1959

14. Leray, J.: Sur le mouvement plan d'un liquide visqueux emplissant l'espace. Acta Math. 63, 193-248 (1934)

15. Orszag, S. A.: Lectures on the statistical theory of turbulence Fluid Dynamics (Summer School, Les Houches, 1973). London: Gordon and Breach (1977) pp. 235-374

16. Ruelle, D.: Differential dynamical systems and the problem of turbulence. Bull. Am. Math. Soc. 5, 29$42(1981)$

17. Scheffer, V.: Turbulence and Hausdorff dimension, In: Turbulence and Navier-Stokes equations, Temam R. (ed.), Lecture Notes in Mathematics, Vol. 565, Berlin, Heidelberg, New York: Springer (1976) pp. $94-112$ 
18. Scheffer, V.: Partial regularity of solutions to the Navier-Stokes equations. Pac. J. Math. 66, 535--552 (1976)

19. Scheffer, V.: Hausdorff measure and the Navier-Stokes equations. Commun. Math. Phys. 55, 97-112 (1977)

20. Scheffer, V.: The Navier-Stokes equations in space dimension four. Commun. Math. Phys. 61, 41-68 (1978)

21. Temam, R.: Navier-Stokes equations, 2nd edn. Amsterdam: North-Holland 1979

22. Vishik, M. L., Fursikov, A. V.: Solutions statistiques homogènes des systèmes paraboliques et du systéme de Navier-Stokes. Ann. Scuola Norm. Pisa, S. IV 4, 531-576 (1977)

23. Vishik, M. L., Fursikov, A. V.: Translationally homogeneous statistical solutions and individual solutions with infinite energy of the system of Navier-Stokes equations. Sib. Math. J. 19, $710-729$ (1978)

Communicated by J. Glimm

Received July 20, 1982; in revised form February 10, 1983 